\title{
Correction to: FLEND (nelarabine, fludarabine, and etoposide) for relapsed T-cell acute lymphoblastic leukemia in children: a report from Japan Children's Cancer Group
}

Tadashi Kumamoto $^{1} \cdot$ Hiroaki Goto $^{2}$. Chitose Ogawa ${ }^{1} \cdot$ Toshinori Hori $^{3} \cdot$ Takao Deguchi $^{4} \cdot$ Takuya Araki $^{5}$. Akiko M. Saito $^{6} \cdot$ Atsushi Manabe $^{7} \cdot$ Keizo Horibe $^{6} \cdot$ Hidemi Toyoda $^{8}$

Published online: 23 January 2021

(c) Japanese Society of Hematology 2021

\section{Correction to: \\ International Journal of Hematology (2020) 112:720-724 \\ https://doi.org/10.1007/s12185-020-02962-2}

In the original publication of the article, Table 1 was incorrectly published. The correct Table 1 is given in this correction.

The original article can be found online at https://doi.org/10.1007/ s12185-020-02962-2.

Tadashi Kumamoto tkumamot@ncc.go.jp

1 Department of Pediatric Oncology, National Cancer Center Hospital, 5-1-1 Tsukiji, Chuo-ku, Tokyo 104-0045, Japan

2 Department of Hematology and Oncology, Children's Cancer Center, Kanagawa Children's Medical Center, Yokohama, Japan

3 Department of Pediatrics, Aich Medical University Hospital, Nagoya, Japan

4 Division of Cancer Immunodiagnostics, Children's Cancer Center, National Center for Child Health and Development, Tokyo, Japan

5 Department of Clinical Pharmacology and Therapeutics, Gunma University Graduate School of Medicine, Maebashi, Gunma, Japan

6 Clinical Research Center, National Hospital Organization Nagoya Medical Center, Nagoya, Japan

7 Department of Pediatrics, Hokkaido University Graduate School of Medicine, Sapporo, Japan

8 Department of Pediatrics, Mie University Graduate School of Medicine, Tsu, Mie, Japan 
Table 1 The study design of FLEND (ALL-RT11)

\begin{tabular}{lllll}
\hline & $\begin{array}{l}\text { Number of } \\
\text { patients }\end{array}$ & $\begin{array}{l}\text { Etoposide } \\
\left(\mathrm{mg} / \mathrm{m}^{2} / \text { day }\right)\end{array}$ & Fludarabine $\left(\mathrm{mg} / \mathrm{m}^{2} /\right.$ day $)$ & Nelarabine $\left(\mathrm{mg} / \mathrm{m}^{2} /\right.$ day $)$ \\
\hline $\begin{array}{l}\text { Administration } \\
\text { Phase } 1^{\mathrm{b}}\end{array}$ & & $1 \mathrm{~h}$ div & $0.5 \mathrm{~h}$ div after ETP & 1 h div $4 \mathrm{~h}$ after FLD \\
Dose level & & & & \\
-2 & $3-6$ & 50 & 20 & 650 \\
-1 & $3-6$ & 75 & 20 & 650 \\
1 & $3-6$ & 75 & 30 & 650 \\
2 & $3-6$ & 100 & 30 & 650 \\
Phase $2^{\mathrm{c}}$ & $22-25$ & RP2D & RP2D & 650 \\
\hline
\end{tabular}

$F L D$ fludarabine, $N E L$ nelarabine, $R P 2 D$ recommended phase 2 dose

${ }^{a}$ All agents were administered for 5 consecutive days

${ }^{b} 3+3$ design, the dose-finding study was started from dose-level 1

'Simon's 2-stage optimal design, div intravenous drip

Publisher's Note Springer Nature remains neutral with regard to jurisdictional claims in published maps and institutional affiliations. 